\title{
Application of Virtual Simulation Technology in Chemistry Teaching
}

\author{
Zhaoyan $\mathrm{Li}^{1,2}$, Yuan $\mathrm{Cao}^{3}$ and Jiancheng $\mathrm{Luo}^{1,2, \text { a }}$ \\ ${ }^{1}$ School of Biological and Chemical Engineering, Nanyang Institute of Technology, Nanyang, Henan, 473004, China \\ ${ }^{2}$ Nanyang Key Lab for Catalytic Functional Materials, Nanyang, Henan, 473004, China \\ ${ }^{3}$ School of Information Engineering, Nanyang Institute of Technology, Nanyang, Henan, 473004, China
}

\begin{abstract}
Nowadays, the application of virtual simulation experiment teaching resources in universities is not systematic and comprehensive enough. Therefore, integrating professional-related virtual simulation experiment teaching resources and constructing virtual simulation comprehensive training courses can not only strengthen and expand professional knowledge and improve professional thinking but also strengthen students' ability to realize innovative thinking. While enriching experimental teaching forms and teaching effects, virtual simulation technology also promotes the integration of experimental teaching and theoretical teaching. The article mainly discusses the application of virtual simulation technology in university chemistry teaching practice and its impact on teaching effects and then summarizes the entry point and expansion form of virtual simulation technology in the curriculum, and serves the promotion of digital and information technology in chemistry teaching application.
\end{abstract}

\section{Introduction}

The integration of theoretical teaching and practical teaching is one of the core issues in chemistry teaching in colleges and universities [1]. The organic combination of the two will help students deepen their understanding of the structure of matter and reaction mechanism. However, due to time and space constraints, chemical experiments and chemical theory teaching are often divided into two parallel teaching courses. Therefore, many students have a separation of mechanism-structure-phenomenon in their learning, and they can only rely on rote learning to learn theories. Knowledge cannot be learned and used lively and truly mastered. With the deepening of information technology in teaching, virtual simulation technology has received more and more attention in chemistry teaching [2-3]. The application of virtual simulation technology in teaching is a digital extension of traditional classrooms. It has the unique advantages of visualization, intuition, and strong immersion. It can also realize some practical content that cannot be carried out due to factors such as time, space, cost, and safety. It has broad application prospects in engineering experimental teaching. In addition to its application in chemical experiment teaching, virtual simulation technology can also compress experiments into the classroom, dynamically present micro-mechanisms, enrich the classroom teaching methods while strengthening the connection between experiment and theory, and stimulate interest in learning at the same time [4-5]. The degree of students' knowledge understanding realizes a virtuous circle from theory to practice and then to theory.

\section{The macroscopic three-dimensional presentation of the microstructure}

In chemistry teaching in colleges and universities, the reaction mechanism is often a difficult point in related chapters. The content of this part is relatively abstract and tests students' ability to think about molecular microstructures. Taking optical isomerism as an example, this chapter involves a large amount of content that requires students to understand the spatial configuration of molecular structures. Traditional teaching relying on textbooks and PPT courseware for flattened teaching often fail to completely transfer the knowledge understood by teachers to students, especially for some For students with weak spatial thinking ability, there are many difficulties in understanding this teaching method. Relying on the threedimensional modeling of virtual simulation technology, the originally abstracted spatial microstructure can be presented at a macro level, allowing students to freely rotate the three-dimensional model, and then have an intuitive understanding of the spatial configuration of the molecular structure, not only according to the rules Judging the basic requirements of the types of optical isomerism, but also an in-depth and intuitive understanding of the nature of this part of the knowledge, which is relatively lacking in traditional teaching programs.

\footnotetext{
${ }^{a}$ Corresponding author: Luojiancheng@nyist.edu.cn
} 


\section{Dynamic presentation of the reaction process}

The chemical reaction is an important content of chemistry teaching in colleges and universities, and the reaction mechanism is the focus and difficulty of this part of the teaching content. In traditional teaching, students are presented with static reaction equations. Taking olefin addition reaction as an example, although the appearance as an electrophilic addition reaction is relatively simple, it involves atomic orbital hybridization, $\pi$-bond electron cloud distribution, induction, and co-existence. The yoke effect and so on. Merely memorizing the rules by rote can cope with the exam, but this method leaves hidden dangers for the subsequent learning of knowledge points. The relationship between the structure and the nature of the response is only a simple memory of the rules. When encountering a complicated process, students cannot correctly grasp the law of reaction. The spatial expression and dynamic expression of the use of virtual simulation technology can make the complex reaction process vivid and organized, and truly connect the thoughts of the teacher and the thoughts of the students. Not only that, through reasonable design, virtual simulation can also make a concrete description of some abstract concepts in the reaction process, such as electron cloud distribution, electron absorption effect, electron donation effect, etc., to deepen students' understanding of abstract concepts. Close to the perspective of scientific facts, understand the essence of the reaction process in a comprehensive and three-dimensional way.

\section{Introduce miniature exquisite experiments into the classroom}

The advantages of virtual simulation technology, such as three-dimensional presentation and dynamics, determine that it has the advantages of intuitiveness, continuity, and organization in the classroom teaching process, which conforms to the cognitive law of students. Besides, virtual simulation experiments also overcome some Chemical experiments that are expensive, time-consuming, and difficult to carry out chemical experiments. Using virtual simulation technology and reasonable instructional design, most of the key steps of the experimental process can be compressed to ten minutes or even less time presented in the classroom. Different from the virtual simulation of chemical experiment teaching, the virtual simulation experiment introduced in the classroom weakens the grasp of the specific experimental process, strengthens the students to explore the relationship between the characteristics, nature, and structure of the reaction from the experimental design, and deepens the students' core knowledge While understanding, enhance the interest and richness of teaching and turn passive teaching into active learning. Taking the properties of solid acid as an example, the core content of this part of the teaching is to enable students to understand the causes of acid sites in solid acids and the relationship between the structure, properties, and catalytic effects of solid acids. In this chapter, the typical solid acid preparation process and solid acid catalysis process can be introduced into classroom teaching in the form of virtual simulation experiments. Teachers use a problem-oriented method to design the teaching process, such as "How to prepare solid acid catalysts to make biodiesel the highest yield" which allows students to play freely in the virtual simulation experiment and put forward the core links in the form of thinking questions. Virtual simulation can allow students to present the expected data results of the experiment in a short period. By analyzing and characterizing the data, students can actively explore the essence of theoretical knowledge and the correlations contained therein. This process has its suitability online The course adopts the mixed teaching mode of the flipped classroom.

\section{The enhancement of online courses by virtual simulation technology}

In recent years, the construction of online courses has greatly promoted the dissemination of education. Unlike classroom teaching, online courses are more flexible and more in line with the fragmented learning habits of students, so they are welcomed by students; students learn basic knowledge through online teaching, and teachers are In the teaching process, more time can be freed up to solve the key and difficult problems of the course, which not only saves teaching time but also has a good interface with new teaching methods such as flipped classrooms. The two key issues in the construction of online courses are the accumulation of resources and course design. The accumulation of resources should focus on curriculum design, present high-quality content in the curriculum, and deepen students' knowledge of the knowledge system. Virtual simulation technology not only breaks the time constraints of some representative experiments but also breaks the constraints of the experiment on the location and environment with the help of network technology. Embedding carefully selected virtual simulation experiments in online courses can further shorten the connection between students' theoretical study and scientific practice, and transform online courses from the supplement and extension of traditional classrooms to students' free exploration and active cognition. Shangyuandi. Chemistry is a practical science. The virtual simulation experiment system connected with online courses provides students with a practical platform to explore anytime and anywhere. This mode is not restricted by the teaching time and teaching environment. This open learning cognitive environment allows students to think about problems independently, verify textbook theories from multiple angles, and realize the learning of theoretical knowledge based on practice rather than rote memorization. This is important to the quality of undergraduate chemistry courses. Promotion is of great significance.

At present, the construction of the virtual simulation experiment system has been paid attention to both from the national policy and the school level. The introduction of virtual simulation technology from experimental teaching into classroom teaching is of unique significance for the curriculum design of chemistry subjects in colleges 
and universities. In the problem-oriented and student-led experimental design exploration, virtual simulation technology promotes the in-depth integration of theoretical knowledge and experiment in chemistry teaching, so that the abstract mechanism is implemented. In this process, students deepen their knowledge. It has established basic scientific research thinking, which enriches the teaching mode and solves the problem of low student participation in traditional classrooms for teachers. The introduction of virtual simulation technology into the classroom is an extension of information technology in chemistry teaching, and it has a good interface with new teaching methods such as online course construction and flipped classrooms.

\section{$6 \mathrm{Key}$ issues and potential solutions in simulation experiments}

\subsection{The simulation experiment software is expensive}

Simulation experiments require high-standard computer equipment to simulate precise phenomena with complete details. The experiments require the creation of a threedimensional virtual laboratory, which requires a large amount of human and financial resources. So far, the price of simulation experiment software is relatively high. When it comes to intellectual property considerations, sufficient funds are required for purchase and subsequent operation updates. Insufficient purchase funds are an important problem for local universities in the construction of simulation laboratories.

(1) Cooperating with universities that have built simulation laboratories, the two parties share the simulation experiment platform to realize the mutual complementation of resources. The Ministry of Education pointed out that the construction of the virtual simulation experiment teaching center must adhere to "scientific planning, sharing resources, highlighting key points, improving efficiency, and sustainable development." The core of the notice is to share high-quality resources, exchange what is needed, and establish a benign, recyclable, and sustainable mechanism among universities. Therefore, we can cooperate and share resources with established universities in chemical simulation experiments, and reduce the high cost of introducing simulations.

(2) Apply for national special fund support. To implement the requirements of the national informatization development strategy, the application of simulation technology in the fields of scientific research, education, and teaching is gradually deepening. In recent years, the Ministry of Education has strengthened its macro-guidance on the informatization of experimental teaching and has successively introduced strong policies and measures. With the support of a good policy environment, universities can establish simulation laboratories based on appropriate investment in construction, and at the same time apply for the support of the national special fund to improve the level and scale of virtual simulation laboratory construction.

\subsection{Simulation experiments cannot completely replace physical experiments}

Although simulation experiments have more unique advantages than traditional experimental teaching methods in chemical experiment teaching, simulation experiments cannot completely replace physical experiments. A simulation experiment is to use computer software to accurately simulate real objects, but it cannot completely restore reality. It simulates every data and reaction accurately and perfectly. However, in real experiments, there will always be certain errors and incomplete responses. It is a place to pay attention to. Therefore, in addition to being familiar with the experimental principles and procedures in the virtual simulation experiment, students also need to go to the chemistry laboratory to conduct physical experiments to truly obtain information and knowledge from the physical experiments. Simulation experiments can be used as a supplement and improvement of the physical experiment, but they cannot replace physical experiments.

\subsection{Simulation experiments hinder the cultivation of students' practical ability}

Virtual simulation experiment does not exist in reality. Students only need to move the mouse to manipulate the experimental knowledge gained from the experiment in the simulation experiment. The experimental knowledge gained from the experiment is relatively shallow, without the depth and completeness of the real experiment, and the practical ability may be lacking in the real experiment. Virtual experiments will also reduce students' perceptual cognition of real objects and affect their practical ability. Therefore, when teaching chemistry experiments, teachers should start from practice, and finally practice, using simulation experiments as tools and means to enhance students' understanding, and ultimately restore the experiments to reality.

\subsection{Lack of simulation experiment researcher}

Simulation experiments require a team of professional programmers and teachers. However, as an emerging project, it appears late and requires a high degree of professionalism. Although many teachers are familiar with the process and principles of physical experiments, they have not been exposed to simulation experiments before. This new teaching method, coupled with the content of chemical simulation, basically involves largescale production processes and processes. It is inherently difficult and there are fewer people familiar with such experimental teaching. Therefore, the lack of relevant teacher resources is more common. This requires the School of Chemistry to start the training and expansion of simulation experiments as soon as possible, and train a group of professionals who teach simulation experiments. 


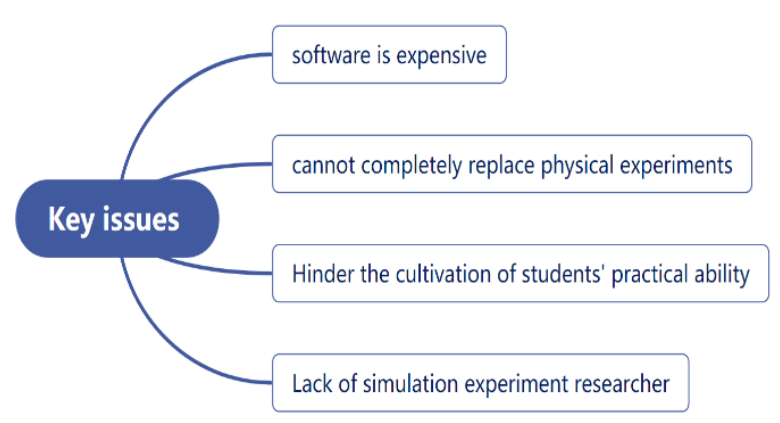

Figure 1. The key issues in simulation experiments.

\section{Prospects of simulation experiments}

The future direction of laboratory construction is technological, interactive, and intelligent. This requires not only continuous progress and development of software, but also resource sharing and resource opening between universities and colleges, and relevant safeguards and Only by improving the relevant management system can the development of the simulation laboratory be accelerated and the related construction of the laboratory can be promoted. Besides, with the rapid development of educational informatization, simulation experiments are gradually moving to deeper and more complex problems while satisfying simple experiments. This requires not only the improvement of the theoretical system of simulation experiments but also continuous Only by absorbing the knowledge and technology of other disciplines can the effect of simulation experiment teaching be improved. Therefore, it is necessary to develop necessary and applicable innovative simulation experiment teaching resources according to the course conditions.

In general, although simulation still has some shortcomings and defects in chemical experiment teaching, it cannot completely replace traditional experiment teaching. However, the advantages of simulation experiments are obvious, with the characteristics of time, interactivity, intelligence, and simulation. The use of software instead of hardware breaks free from the limitations of many objective factors on chemical experiments, optimizes the experimental teaching environment, and creates a diverse teaching environment Enhance the interaction between experiment and teaching, create a safer experimental teaching atmosphere, and at the same time stimulate students' interest in learning and the development of innovative thinking, which has an irreplaceable effect on reforming and innovating chemical experimental teaching mode and improving students' scientific literacy. Through the construction of simulation laboratories, we will further promote the integration of teaching resources and information technology, promote the innovation and modernization of experimental methods and experimental methods, innovate talent training models, and comprehensively improve the quantity and quality of talent training.

\section{References}

1. Ahamed, H. H. O. . (2014). Implementation of virtual laboratories in teaching practical general chemistry.

2. Ali, N. , Ullah, S., Alam, A. , \& Rafique, J. . (2014). 3D Interactive Virtual Chemistry Laboratory for Simulation of High School Experiments. International Conference on Computer Graphics, Animation and Gaming Technologies (EURASIA Turkey 2014).

3. Belletti, A., Borromei, R. , \& Ingletto, G. . (2006). Teaching physical chemistry experiments with a computer simulation by labview. Journal of Chemical Education, 83(9), 1353-1355.

4. Beal, C. R., \& Stevens, R. H. . (2011). Improving students' problem solving in a virtual chemistry simulation through metacognitive messages. Society for Research on Educational Effectiveness, 5.

5. Lihua, L., Jing, C., Xiaoqun, L., \& Anxin, Z. . (2013). Research and implementation of virtools based on 3D virtual teaching laboratory. 2013 3rd International Conference on Computer Science and Network Technology (ICCSNT). IEEE. 